\title{
Analysis of Blood Group Discrepancy in Healthy Blood Donors at a Tertiary Care Referral Hospital from Eastern India: A Retrospective Study
}

\author{
Ansuman Sahu ${ }^{1}$ Satya Prakash ${ }^{1}$ Niladri Das ${ }^{1}$ Suman Sudha Routray ${ }^{1}$ Archana Naik ${ }^{1}$ \\ Somnath Mukherjee ${ }^{10}$ \\ ${ }^{1}$ Department of Transfusion Medicine, All India Institute of Medical \\ Sciences, Bhubaneswar, Odisha, India \\ Address for correspondence Somnath Mukherjee, MD, Additional \\ Professor, Department of Transfusion Medicine, All India Institute of \\ Medical Sciences, Bhubaneswar, 751019, Odisha, India \\ J Lab Physicians 2022;14:247-252. \\ (e-mail: som.mukherjee2011@gmail.com).
}

\begin{abstract}
Keywords

- blood donors

- ABO blood-group system

- ABO antibodies

- adsorption-elution studies

Objective $A B O$ typing constitutes cell grouping and serum grouping. The discrepancy may arise in $A B O$ typing due to a mismatch in cell grouping and serum grouping. It may be due to technical errors, missing or weak $A B O$ antibodies (type I), weak $A B O$ subgroups (type II), Rouleaux formation (type III), or other miscellaneous reasons (type IV). This study was carried out to determine the prevalence and cause of ABO blood group discrepancy in donor samples at our center.

Methods A retrospective study of ABO blood group typing of blood donors was conducted at our center. The blood group typing was routinely performed using gel cards and a microcentrifuge system (Tulip Diagnostics(P) Ltd, Goa, India). If any discrepancy in $A B O$ typing was noted, the test was repeated using the conventional tube technique. After sorting clerical/technical error, the causes of discrepancy were analyzed and resolved using anti- $\mathrm{A}_{1}$, anti- $\mathrm{H}$, anti-AB, and other immunohematological tests like antibody screening and identification, saliva inhibition test, adsorptionelution studies.

Results A total of 12,715 (98.6\% males and 1.4\% females) donor samples were tested. The number of $A B O$ discrepancies detected were 15 (0.12\%). The discrepancies were characterized as type I ( 6 cases; $40 \%$ ), type II ( 1 case; $6.7 \%$ ), type III ( 0 cases; $0 \%$ ), and type IV (8 cases; $53.3 \%$ ). Three cases, each of anti-M and anti-Le ${ }^{\mathrm{b}}$, were detected in the study population. A single case of $A_{3}$, a subgroup of $A$ blood group, was found during the study.

Conclusion The prevalence of $A B O$ group discrepancy was $0.12 \%$ at our center. Discrepancy arising during $A B O$ typing of blood donor must be resolved before reporting $A B O$ blood group to minimize the recipient's chances of transfusion reaction. The serum grouping is equally crucial as cell grouping for reporting the $A B O$ group of an individual.
\end{abstract}

published online February 9, 2022
DOI https://doi.org/

$10.1055 / \mathrm{s}-0042-1742417$. ISSN 0974-2727.
(C) 2022. The Indian Association of Laboratory Physicians. All rights reserved.

This is an open access article published by Thieme under the terms of the Creative Commons Attribution-NonDerivative-NonCommercial-License, permitting copying and reproduction so long as the original work is given appropriate credit. Contents may not be used for commercial purposes, or adapted, remixed, transformed or built upon. (https://creativecommons.org/ licenses/by-nc-nd/4.0/)

Thieme Medical and Scientific Publishers Pvt. Ltd., A-12, 2nd Floor, Sector 2, Noida-201301 UP, India 


\section{Introduction}

The ABO blood-group system discovery by Karl Landsteiner has become a major landmark as it heralded a significant revolution in the field of transfusion medicine. ABO blood grouping is one of the most common blood group systems that can be performed by simple technique and it involves two basic steps: cell grouping (forward grouping) that establishes the presence or absence of A and/or B antigens on the red blood cells (RBCs) and serum grouping (reverse grouping) that demonstrates the presence or absence of $\mathrm{ABO}$ antibodies in the serum. ${ }^{1,2}$ The naturally occurring $\mathrm{ABO}$ antibodies comprise of mainly anti-A and anti-B that are predominantly immunoglobulin $M$ type and generally detectable by the age of 6 months. $^{3}$ Determination of correct $\mathrm{ABO}$ blood grouping needs both cell grouping and serum grouping because of the existence of a mutual association between cell grouping and serum grouping. ${ }^{2}$ These ABO cell and serum groupings should be done in both donor and patient to correctly assign the blood group. Correct assignment of blood group not only prevents $A B O$ mismatch blood transfusion but also helps in providing correct blood product in $\mathrm{ABO}$ mismatch organ transplant and prevents organ rejection. ${ }^{4}$ However, blood group discrepancies still occur during routine blood transfusion practices. Blood group discrepancies are usually encountered when there is a deviation from the expected pattern of antigen in cell grouping and antibody in serum grouping. These blood group discrepancies occur due to weaker expression of $A$ or $B$ antigen or missing or low titer of ABO antibodies. Other reasons may be related to technical error or clerical error, miscellaneous, or rouleaux formation, which gives rise to the blood group discrepancy. ${ }^{2,5}$ As per the standard classification of ABO discrepancy, blood group discrepancy is divided into four major categories: type I, type II, type III, and type IV ABO blood group discrepancy. ${ }^{6}$ Any blood group discrepancy needs immediate attention to resolve so that the correct blood group could be assigned to the donor or patient and transfusion error due to mismatch blood transfusion could be prevented. Although there are few pieces of literature on blood group discrepancy published from our country, there are hardly any data available on donor blood group discrepancy from the eastern part of our country. The most possible reason could be that the many blood centers in our region still do not perform reverse or serum blood grouping and the majority of the blood centers still do not perform antibody screening of the blood donors. These irregular red cell antibodies in donors can seldom cause severe transfusion reaction in pediatric population or in cases of massive transfusion when a large volume of plasma is transfused. ${ }^{7}$ Therefore, we intended to perform a retrospective analysis on blood group discrepancy among blood donors as we encountered blood group discrepancies while performing routine reverse grouping or while performing antibody screening of the donor. The main purpose was to determine the prevalence and cause of $\mathrm{ABO}$ blood group discrepancy in donor samples in our center.

\section{Methodology}

This retrospective study was conducted from January 2019 to July 2020 in the Department of Transfusion Medicine at a tertiary care referral hospital from Eastern India. The study was approved by Institutional Ethics Committee with the approval letter no: T/IM-NF/Trans.Med/20/109. There were 12,715 donors including 12,534 (98.6\%) male and $181(1.4 \%)$ female. The deferred donors were excluded from the study. Detailed demographic data of the donors were collected from e-Raktokosh software installed in the department. ABO blood group typing results were analyzed from the donor blood grouping register and blood grouping discrepancy register.

During whole blood donation, samples were collected from the diversion sample pouch containing a Leur adapter for routine ABO typing and TTI testing in Ethylenediaminetetraacetic acid and plain vials. Donor samples were tested for $\mathrm{ABO}$ typing using Matrix forward and reverse grouping card by column agglutination technique (Tulip Diagnostics (P) Ltd, Goa, India) as per departmental standard operating procedure. Antibody screening with in-house prepared pooled $O$ cell was performed after routine ABO typing in donor sample. Whenever there is a discrepancy between forwarding grouping and reverse grouping, technical/clerical errors were sorted. ABO typing test was repeated on the same sample using the same column agglutination technique and conventional tube technique (CTT). After ruling out technical/clerical errors, problems with RBCs or plasma were studied. Monoclonal antisera anti-A, anti-B, anti-AB, anti-H manufactured by Tulip Diagnostics (P) Ltd, Goa, India, were used for forward grouping and in-house pooled A cell, B cell, and $O$ cell are used for reverse grouping for CTT for resolution of $\mathrm{ABO}$ grouping discrepancy. In case of missing or weak antibodies, the incubation time was prolonged at room temperature, or incubation at $4^{\circ} \mathrm{C}$ was performed; still, if the discrepancy was not resolved, saliva inhibition testing and adsorption elution test were done to confirm the grouping. When there was an agglutination with "O" cell either in gel card or CTT, the antibody screening (AS) procedure was performed using three-cell antigen panel ID-DiaCell I-II-III (DiaMed GmbH, BIO-RAD, Switzerland). 11-cell antibody panel, ID- Panel (DiaMed GmbH, BIO-RAD, Switzerland), and select cells were used for antibody identification. Details of workflow for resolution of the discrepancy are depicted in -Fig. 1 All the data were entered into MS Excel (Microsoft Inc., Redmond, Washington, USA) spreadsheet. Various calculations, for example, incidence, prevalence, and rate, were calculated using standard formulae using MS Excel.

\section{Results}

A total of 12,715 donors were studied for ABO typing results, including 12,534 (98.58\%) males and 181 (1.42\%) females. $\mathrm{ABO}$ blood groups among the donors were distributed as $\mathrm{A}$ 21.9\%, B 32.5\%, 0 38.6\%, and $A B$ 7\%. After sorting clerical error, $15(0.12 \%)$ numbers $A B O$ blood group discrepancies are found. All these donors were male, having a mean age of 32.7 


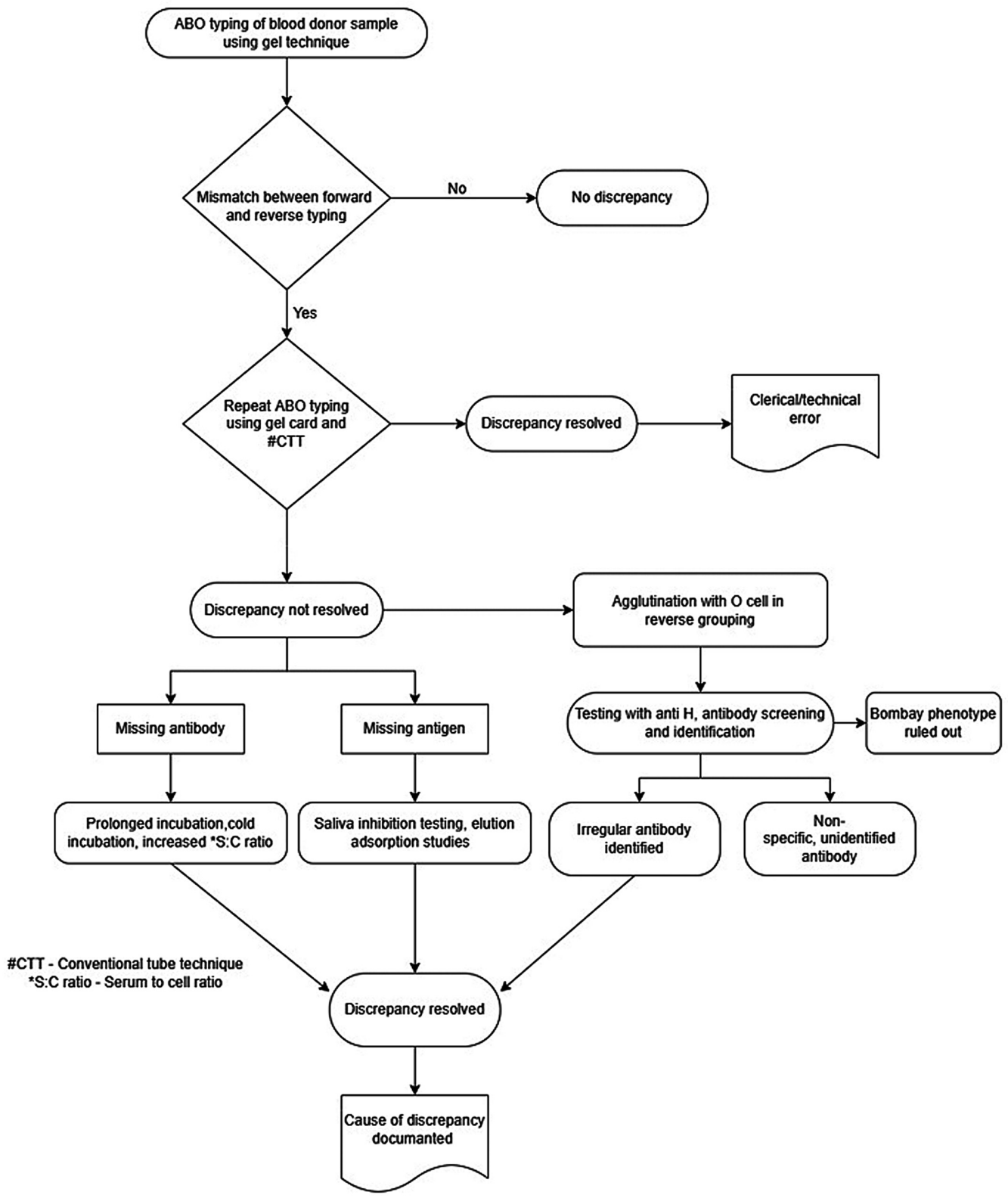

Fig. 1 Details of workflow for resolution of the discrepancy.

Table 1 Summary of ABO discrepancies

\begin{tabular}{|l|l|l|l|}
\hline $\begin{array}{l}\text { Type of } \\
\text { blood group } \\
\text { discrepancy }\end{array}$ & Cause & $\begin{array}{l}\text { Total number } \\
\text { of discrepancy (\%) }\end{array}$ & Remarks \\
\hline Type I & Weak or missing antibodies & $6(40 \%)$ & 4 weak anti-B and 2 weak anti-A \\
\hline Type II & Weak or missing antigen & $1(6.67 \%)$ & $\mathrm{A}_{3}$ \\
\hline Type III & Rouleaux formation & $0(0 \%)$ & 0 \\
\hline Type IV & $\begin{array}{l}\text { Miscellaneous or } \\
\text { irregular antibody }\end{array}$ & $8(53.33 \%)$ & 3 anti-M, 3 anti-Le ${ }^{\text {b }}$ and 2 unidentified \\
\hline
\end{tabular}

years. The discrepancies were classified as type I to type IV as per - Table 1. Type IV discrepancies were maximum in this study, for example, 8 (53.33\%) followed by type I $(6 ; 40 \%)$ and type II discrepancies $(1 ; 6.67 \%)$. There were no type III discrepancies found during the study period. The only type II discrepancy was possibly an $A_{3}$, a subgroup of the A blood group. Serological characteristics of $A_{3}$ have been shown in - Table 2. Type I discrepancies comprised of four weak anti-B 
Table 2 Serological details of weak subgroups of A $(n=1)$

\begin{tabular}{|c|c|c|c|c|c|c|c|c|c|c|}
\hline \multicolumn{4}{|c|}{ Cell grouping } & \multicolumn{3}{|c|}{ Serum grouping } & \multirow[t]{2}{*}{ Auto control } & \multirow[t]{2}{*}{ Anti-H lectin } & \multirow{2}{*}{$\begin{array}{l}\text { Anti- } A_{1} \\
\text { lectin }\end{array}$} & \multirow{2}{*}{$\begin{array}{l}\text { Possible } \\
\text { weak subgroup }\end{array}$} \\
\hline Anti-A & Anti-B & Anti-D & Anti-AB & A cell & B cell & $\mathrm{O}$ cell & & & & \\
\hline $\begin{array}{l}2+1 \\
\mathrm{Mf}^{\mathrm{a}}\end{array}$ & 0 & $4+$ & $2+$ & 0 & $4+$ & 0 & 0 & $4+$ & 0 & $A_{3}$ \\
\hline
\end{tabular}

${ }^{\mathrm{a} M f}$, mixed field.

antibodies and two weak anti-A antibodies. In type IV discrepancy, three anti-M and three anti-Le ${ }^{\mathrm{b}}$ were identified. The other two type IV discrepancies could not be resolved by the available screening and identification cell panel. A maximum number of discrepancies were found in A blood group donor having an incidence of $0.05 \%$, followed by $\mathrm{O}$ blood group donors having the same of $0.04 \%$.

\section{Discussion}

In this retrospective study, we observed 15 cases of $A B O$ discrepancy with an overall prevalence of $0.12 \%$. Similar studies done by other authors from different parts of our country ( - Table 3 ) have shown that the prevalence of ABO discrepancy ranges from 0.02 to $0.064 \%{ }^{1,2,4,8}$ We observed a little higher prevalence rate of $A B O$ discrepancy in comparison to the other studies. Genetic diversity along with a demographic variation of the population in this part of the country could be a possible explanation of the different prevalence rate of $\mathrm{ABO}$ discrepancy. Comparatively lesser population sample size in our study could also be another reason for a higher prevalence rate of $A B O$ discrepancy. We also found type IV discrepancy to be the most common $A B O$ blood group discrepancy that constituted $53.33 \%$ (8 cases) of total ABO discrepancy. In type IV discrepancy, there is unusual or unexpected reactivity in the reverse grouping showing positive agglutination with the reagent $O$ cells. Type IV discrepancy is usually attributed to the presence of cold reactive alloantibodies or cold reactive isoantibodies or cold reactive autoantibodies present in the serum of the donor or patient sample. ${ }^{6,9}$ Out of eight cases, three donors had naturally occurring irregular antibody with the specificity of anti-M and three donors had antibody specificity of anti-
$\mathrm{Le}^{\mathrm{b}}$ as they were reactive with $\mathrm{O}$ cells at room temperature. However, the thermal amplitude of these two antibodies extended up to $37^{\circ} \mathrm{C}$ degrees and was therefore clinically significant. A similar result was reported by other studies which found that the antibodies against the MNS blood group system are the most common irregular antibodies in the blood donors. ${ }^{710}$ Rest two cases we could not determine antibody specificity. This is likely because the commercially available red cell panel was typed only for major clinically significant red cell antigens and the antibody formed in these donors may be directed to red cell antigen but not mentioned in the screening and identification red cell panel chart. A similar study was done by N Garg et al also explained alike possibilities for inconclusive results with positive antibody screening. Another explanation the author cited that the antibodies could be in low titers or the developing phase. ${ }^{7}$

We encountered six cases of type I discrepancy that constituted $40 \%$ of total $\mathrm{ABO}$ discrepancy (-Table 1). This is the second most common type of blood group discrepancy we observed in our study. Type I discrepancy characterizes by a diminished reaction in reverse grouping due to missing or weak antibodies. ${ }^{9,11}$ Out of six cases, four donor samples had blood group discrepancy due to weak $B$ antibodies, and the rest two samples had both anti-A and anti-B antibodies. The presence of weak $B$ antibody is more common than the presence of anti-A antibody in type I ABO discrepancy. A recent study done by Jain et al and a study by Sharma et al observed that type I ABO discrepancy accounted for the predominant type of $A B O$ blood group discrepancy with a percentage frequency of 35.5 and $58.8 \%$, respectively. ${ }^{4,8}$ Moreover, both the studies found that weak or low avidity anti-B antibody is the most common cause of type I ABO blood group discrepancy. The changes of $A B O$ isoagglutinin

Table 3 Studies on blood group discrepancies in donor samples

\begin{tabular}{|c|c|c|c|c|c|c|}
\hline Author & Year & Sample size & $\begin{array}{l}\text { Number and } \\
\text { prevalence } \\
\text { of } A B O \\
\text { discrepancies }\end{array}$ & $\begin{array}{l}\text { Prevalence of } \\
\text { weak subgroups }\end{array}$ & Rate of weak subgroups of $A$ & Rate of weak subgroups of $B$ \\
\hline Thakral et al $^{13}$ & 2005 & 86,687 & Not determined & $0.02 \%$ & 1 in 43,344 to 1 in 14,448 & 1 in 86,687 \\
\hline Sharma et $\mathrm{al}^{8}$ & 2013 & 104,010 & $51 ; 0.04 \%$ & $0.01 \%$ & Not determined & Not determined \\
\hline Kaur et $\mathrm{al}^{2}$ & 2013 & 44,425 & $28 ; 0.06 \%$ & $0.04 \%$ & 1 in 11,106 to 1 in 44,425 & $\begin{array}{l}1 \text { in } 22,212 \text { to } 1 \\
\text { in } 44,425\end{array}$ \\
\hline Makroo et al ${ }^{1}$ & 2019 & 62,080 & $14 ; 0.02 \%$ & $\begin{array}{l}0.002 \% \\
\text { (only subgroup } \\
\text { of } A \text { ) }\end{array}$ & 1 in 13,025 & None \\
\hline Jain et $\mathrm{al}^{4}$ & 2018 & $1,44,279$ & $93 ; 0.064 \%$ & $0.02 \%$ & 1 in 6,011 & 1 in 28,856 \\
\hline Present study & 2020 & 12,715 & $15 ; 0.12 \%$ & 0.007 & 1 in 12,715 & None \\
\hline
\end{tabular}


levels related to age, immunodeficiency conditions, and other clinical conditions like post-hematopoietic stem cell transplant are few possible causes of weak or missing reactivity of antibodies in the reverse grouping. Therefore, proper review of medical records along with the elicitation of any drug history may assist in the evaluation of such discrepancies.

Type II ABO discrepancy occurs because of an unpredicted reaction in cell grouping owing to diminished expression of the red cell antigens. ${ }^{9,11}$ A weak subgroup of $A$ is more common than a weak subgroup of B. Subgroups of A weaker than $A 2$ contribute to almost $1 \%$ of $A B O$ blood group discrepancy. $^{3}$ The subgroup of A can be broadly divided into two categories depending on the agglutination of red cells with anti-A. $A_{3}, A_{\text {end }}$, and $A_{x}$ are agglutinated with anti-A, while $A_{m}, A_{y}$, and $A_{e l}$ are not. ${ }^{2}$ In our study, we observed only one case $(6.67 \%, 1$ out of total 15 cases) of type II ABO discrepancy which was due to a weak subgroup of A. Study done by Kaur et al and another recent study from Iran reported that subgroups of $A$ antigen account for a most common cause for blood group discrepancy. ${ }^{2,12}$ However, Makroo et al and Jain et al reported that subgroups of A antigen were the second most common cause that contributed to 29 and $25.8 \%$, respectively. ${ }^{1,4} \mathrm{~A}$ small proportion of A population contains subgroups of $A$ antigen. These subgroups have fewer antigen sites on the RBC that result in weakened or even missing reactions in forward grouping. Extended incubation at room temperature or at $4^{\circ} \mathrm{C}$ temperature may augment the reactivity. Testing with monoclonal antibodies like anti-A, $B$ - anti- $A_{1}$, anti-H lectin; testing for $A, B$, and $H$ substances in the saliva of secretors along with adsorption Elution test could be helpful in the determination of blood group. Further, family studies and DNA-based molecular studies are used for final confirmation and determination of the blood group. ${ }^{12}$ The frequencies of different subgroups of A phenotype usually vary with different ethnic groups. As per the studies done from different parts of the world, the $\mathrm{A}_{3}$ subgroup phenotype is the most common among all the weak A subgroups with an estimated frequency varying from 1 in 1,000 group $A$ to 1 in $90,000 .{ }^{13-15}$ In our study, the $A$ subgroup was possibly $A_{3}$ type (Table 2 ) as we observed weak mixed field $(2+)$ reaction in cell grouping with anti-A and anti-AB antisera and strong reaction $(4+)$ with $B$ cell in the reverse grouping. Further testing with anti- $\mathrm{A}_{1}$ lectin showed no reaction but had a strong reaction with anti-H lectin. We performed an adsorption elution test and saliva inhibition test that confirmed the presence of A substance in the donor. However, further molecular study for final confirmation of the $A_{3}$ blood group subtype could not be performed. The frequency of $A_{3}$ subgroup in our study ( 1 in 12,715) was also comparable with the studies reported by Thakral et al ( 1 in $14,448)$ and Kaur et al $(1$ in 11,106$))^{2,13}$ We did not observe any weak $B$ subgroup that is perhaps because the subgroup of $B$ is much less common than the subgroup of $A$ as mentioned in the previous studies. ${ }^{4,12}$

Type III ABO blood group discrepancies occur due to rouleaux formation when there are elevated levels of plasma proteins in certain disease conditions such as multiple myeloma, Waldenstrom's macroglobulinemia, and advanced cases of Hodgkin's lymphoma. Sometimes administration of plasma expanders like dextran and cord blood samples containing Wharton's jelly also give rise to the rouleaux formation or pseudo agglutination. ${ }^{1}$ We did not detect any cases of type III blood group discrepancy, which is possible because type III discrepancy is mostly encountered in patients with certain disease conditions and it is rarely seen in the blood donor population.

Our study was not without limitations; first, our study is a retrospective study and with less sample size compared with other studies. Second, our study performed antibody screening of the blood donors with in-house prepared pooled $\mathrm{O}$ cells. Thus, we might have missed in picking up few irregular alloantibodies in comparison to the commercially prepared screening $\mathrm{O}$ cells that contain most of the clinically significant red cell antigens. Lastly, we did not perform molecular testing for final confirmation of the ABO blood group due to lack of infrastructure at our center. However, this study could be an eye-opener for planning to conduct a study on a larger sample size that will help in identifying any possible clinically significant antibodies in the blood donor. Besides, our study result will also encourage the other blood centers in this region to initiate the routine practice of performing reverse blood grouping as well as antibody screening of blood donors.

\section{Conclusions}

As far as we are aware, this is possibly the first study on donor population from this region where $\mathrm{ABO}$ blood group discrepancy has been identified as per standard classification. The overall prevalence of $\mathrm{ABO}$ blood group discrepancy in our donor population is $0.12 \%$. It is always important to recognize blood group discrepancies and resolve them at the earliest. It is also imperative to identify the weak subgroup of A or B as it helps prevent mistyping the donor unit. Identification of irregular antibodies in the donor unit is another significant aspect as it may have adverse consequences such as hemolytic reaction to the recipient. However, the serologically determined weak subgroups need molecular analysis for further confirmation. The genomic analysis may also unravel some novel mutations that may give rise to new blood group phenotypes in our population.

\section{Funding \\ None.}

Conflict of Interest

The authors declare no conflict of interest.

\section{References}

1 Makroo RN, Kakkar B, Agrawal S, Chowdhry M, Prakash B, Karna P. Retrospective analysis of forward and reverse $\mathrm{ABO}$ typing discrepancies among patients and blood donors in a tertiary care hospital. Transfus Med 2019;29(02):103-109 
2 Kaur G, Kaur P, Basu S, Kaur R. Blood group discrepancies at a tertiary care centre - analysis and resolution. Int J Lab Hematol 2014;36(04):481-487

3 Storry JR, Olsson ML. The ABO blood group system revisited: a review and update. Immunohematology 2009;25(02):48-59

4 Jain A, Garg S, Marwaha N, Sharma RR. ABO blood grouping discrepancies in the donor population. ISBT Sci Ser 2020;15(02):281-285

5 Chiaroni J, Legrand D, Dettori I, Ferrera V. Analysis of ABO discrepancies occurring in 35 French hospitals. Transfusion 2004;44(06):860-864

6 Harmening DM. Modern Blood Banking and Transfusion Practices. 6th edition. Philadelphia, PA: FA Davis Company Publications; 2012

7 Garg N, Sharma T, Singh B. Prevalence of irregular red blood cell antibodies among healthy blood donors in Delhi population. Transfus Apheresis Sci 2014;50(03):415-417

8 Sharma T, Garg N, Singh B. ABO blood group discrepancies among blood donors in Regional Blood Transfusion Centre GTB Hospital, Delhi, India. Transfus Apheresis Sci 2014;50(01):75-80
9 Meny GM. Recognizing and resolving ABO discrepancies. Immunohematology 2017;33(02):76-81

10 Solanki A, Chandra T, Singh A. Prevalence of red blood cell antibodies in whole blood donors: a single-centre experience in north India. Indian J Med Res 2020;152(03):280-284

11 Nepal B, Shrestha B, Maharjan S, Bhasima S, Shrestha SK. ABO blood group discrepancies: Study of prevalence and related factors. Gd Med J 2019;1(02):88-91

12 Javadzadeh Shahshahani H, Hayati A. Blood group discrepancies at a regional blood center. Int J Hematol Oncol Stem Cell Res 2020; 14(01):38-44

13 Thakral B, Saluja K, Bajpai M, Sharma RR, Marwaha N. Importance of weak ABO subgroups. Lab Med 2005;36(01):32-34

14 Reed TE. The frequency and nature of blood group A3. Transfusion 1964;4(06):457-460

15 Garretta M, Muller A, Gener J, Matte C, Moullec J. Reliability in automatic determination of the $\mathrm{ABO}$ group by the groupamatic system. Vox Sang 1974;27(02):141-155 